\title{
MINIMUM TOPOLOGICAL GENUS OF COMPACT BORDERED KLEIN SURFACES ADMITTING A PRIME-POWER AUTOMORPHISM
}

\author{
by E. BUJALANCE,* J. M. GAMBOA** and C. MACLACHLAN***
}

(Received 6 December, 1993; revised 15 August, 1994)

Introduction. In the nineteenth century, Hurwitz [8] and Wiman [14] obtained bounds for the order of the automorphism group and the order of each automorphism of an orientable and unbordered compact Klein surface (i.e., a compact Riemann surface) of topological genus $g \geq 2$. The corresponding results of bordered surfaces are due to May, [11], [12]. These may be considered as particular cases of the general problem of finding the minimum topological genus of a surface for which a given finite group $G$ is a group of automorphisms. This problem was solved for cyclic and abelian $G$ by Harvey [7] and Maclachlan [10], respectively, in the case of Riemann surfaces and by Bujalance [2], Hall [6] and Gromadzki [5] in the case of non-orientable and unbordered Klein surfaces. In dealing with bordered Klein surfaces, the algebraic genus-i.e., the topological genus of the canonical double covering, (see Alling-Greenleaf [1])-was minimized by BujalanceEtayo-Gamboa-Martens [3] in the case where $G$ is cyclic and by McCullough [13] in the abelian case.

A more involved question is to minimize the topological genus when the number of connected components of the boundary is fixed. Our interest in this problem comes from the field of real algebraic geometry. In fact, via the well known equivalence between the categories of compact bordered Klein surfaces and formally real algebraic function fields in one variable over the field $\mathbf{R}$ of real numbers, the problem we are interested in is the following: given a finite group $G$ and a positive integer $k$, to compute the minimum genus of the projective, smooth, irreducible real algebraic curves with $k$ connected components admitting $G$ as a group of birational automorphisms. In this paper we solve the problem when $G$ is a cyclic group of prime-power order (the case of prime order was studied in [4]). Note that for complex curves, $k=1$, and this explains the differences-see the example at the end of the paper-of the behaviour of minimum genus between complex and real curves, when the number of components of the last one is fixed.

The results split into a number of cases depending on whether the surface is orientable or not and whether the automorphism preserves the orientation or not. To simplify the description, we use the notation in [4] which we now briefly expound.

Let $K$ be the class of compact and bordered Klein surfaces. Given $S \in K$, we denote by $g(S)$ its topological genus, $k(S)$ the number of connected components of its boundary and define $\alpha(S)$ to be 2 if $S$ orientable and 1 if $S$ is non orientable. Then, the algebraic genus $p(S)$ of $S$ is given by $p(S)=\alpha(S) g(S)+k(S)-1$ and so, minimizing $g$, for fixed $k$, is equivalent to minimizing $p$. More precisely, let us fix the integers $N \geq 2, k \geq 1$. We denote by $K_{+}^{+}(N, k)$-resp. $K_{+}^{-}(N, k)$-the collection of those orientable surfaces $S$ in $K$

* Partially supported by DGICYT PB 92-0716 and CEE-CHRX-CT93-0408.

** Partially supported by DGICTY PB 92-0498-0498-C02-02 and CEE-CHRX-CT93-0408.

*** The author is grateful to the UNED for an invitation to Madrid, where this work was initiated.

Glasgow Math. J. 37 (1995) 221-232. 
with $p(S) \geq 2, k(S)=k$, which admit an orientation-preserving (resp. orientationreversing) automorphism of order $N$. We also denote by $K_{-}(N, k)$ the family of non-orientable surfaces $S$ in $K$ with $p(S) \geq 2, k(S)=k$, which admit an automorphism of order $N$. Define the numbers

$$
\begin{gathered}
p_{+}^{+}(N, k)=\min \left\{p(S): S \in K_{+}^{+}(N, k)\right\} \quad p_{+}^{-}=\min \left\{p(S): S \in K_{+}^{-}(N, k)\right\} \\
p_{-}(N, k)=\min \left\{p(S): S \in K_{-}(N, k)\right\} .
\end{gathered}
$$

These numbers were computed in [4; Cor 3.2.17] for prime $N$, as a function of $N$ and $k$. In this article we calculate them in the case $N=p^{e}$ is a power of a prime number $p$. Of course, if $g_{+}^{+}(N, k), g_{+}^{-}(N, k)$ and $g_{-}(N, k)$ have the same meaning for the topological genus, one obtains

$$
g_{+}^{+}(N, k)=\frac{1}{2}\left[p_{+}^{+}(N, k)+1-k\right] ; \quad g_{+}^{-}(N, k)=\frac{1}{2}\left[p_{+}^{-}(N, k)+1-k\right]
$$

and $g_{-}(N, k)=p_{-}(N, k)+1-k$, which solves the problem stated above.

The proofs throughout the paper are quite technical but we can summarize the general strategy as follows: let $f$ be an automorphism of finite order $N$ on a surface $S$ whose boundary has $k$ connected components. Let $g^{\prime}$ and $k^{\prime}$ be, respectively, the genus and number of boundary components of the quotient $S^{\prime}=S /\langle f\rangle$. The canonical projection $S \rightarrow S^{\prime}$ ramifies over inner points with ramification indices $m_{1}, \ldots, m_{r}$ and the Riemann Hurwitz formula provides us with a relation:

$$
p(S)=P\left(g^{\prime}, k^{\prime}, m_{1}, \ldots, m_{r}\right)
$$

We get also another relation which expresses essentially how the boundary components of $S$ are mapped to the ones of $S^{\prime}$. This is, roughly speaking, the content of Theorems 3.1.5, 3.1.6, 3.1.8, 3.1.9 and 3.2.3 in [4] which give us the precise function $P$ above and the exact relations satisfied by $N, k, g^{\prime}, k^{\prime}, m_{1}, \ldots, m_{r}$-see e.g. conditions (2.1)-(2.4) in Section 2. The variables $m_{1}, \ldots, m_{r}$ are divisors of $N$ and this is why the case $N=p^{e}$ for prime $p$ is more accessible.

In this way the problem is reduced to one of a combinatorial or arithmetic nature: to get the minimum value of the function $p(S)=P\left(g^{\prime}, k^{\prime}, m_{1}, \ldots, m_{r}\right)$ where the variables run over subsets of non-negative integers and satisfy certain arithmetic relations. To deal with this question we devote the first section of the paper to some arithmetic lemmas, and the next ones to minimizing the different functions $P$ occurring according to the prime $p$ being 2 or not or the orientability character of the input data. Of course, when we calculate the minimum value of $P$ we get also the topological data of the covering $S \rightarrow S^{\prime}$ for which it is attained.

1. " $p$-adic" expansions. For the cyclic group of order $p^{e}$, the results are expressed in terms of a truncated $p$-adic expansion of $k$, the number of boundary components. This type of expansion was used in the related but distinct problem of determining the genera of all compact Riemann surfaces on which a cyclic group of order $p^{e}$ acts, dealt with by Kulkarni and Maclachlan in [9]. 
Given $k$ and $p^{e}$, there exist unique non-negative integers $a_{j}=a_{j}(p, e, k), j=$ $0,1, \ldots, e$ such that

$$
k=\sum_{j=0}^{e} a_{j} p^{j} \quad \text { where } \quad 0 \leq a_{j}<p(j=0, \ldots, e-1), \quad a_{e} \geq 0 .
$$

Define $\rho(p, e, k)=\sum_{j=0}^{e} a_{j}(p, e, k)$.

In the case where $p=2, e>1$, there is also a unique expansion

$$
k=\sum_{j=0}^{e-1} b_{j}(e, k) 2^{j} \quad \text { where } \quad 0 \leq b_{j}(e, k) \leq 1(j=0, \ldots, e-2) \quad \text { and } \quad b_{e-1}(e, k) \geq 0
$$

Define $\varepsilon(e, k)=b_{e-1}(e, k)+2 \sum_{j=0}^{e-2} b_{j}(e, k)$.

Our results will be expressed in terms of these functions $\rho(p, e, k)$ and $\varepsilon(e, k)$, since the expressions for $k$ given above are "minimal" in a sense appropriate for our calculations. The basic lemma expressing this "minimality" is

LEMMA 1.1. Let $p$ denote a prime number, and $k, e$ positive integers. Then, for integers $y_{0}, y_{1}, \ldots, y_{e},\left(y_{i} \geq 0\right)$ such that $k=\sum_{i=0}^{e} y_{i} p^{e-i}$ the following conditions hold:

(1) $\sum_{i=0}^{e} y_{i} \geq \rho(p, e, k)$.

(2) If $y_{r} \neq a_{e-i}(p, e, k)$ for some $0 \leq i \leq e$, then $\sum_{i=0}^{e} y_{i}-\rho(p, e, k) \geq p-1$.

(3) If $p=2$, then $\sum_{i=0}^{e} y_{i}-\rho(2, e, k) \geq \frac{\left(y_{1}-a_{e-1}\right)}{2}$.

(4) If $p=2, e>1, k$ is multiple of 4 and $y_{e} \geq 2$, then $\sum_{i=0}^{e} y_{i}-\rho(2, e, k) \geq 2$.

Proof. For $y=\left(y_{0}, y_{1}, \ldots, y_{e}\right)$ as in the statement, define

$$
\delta_{e}(y, k)=\sum_{i=0}^{e} y_{i}-\rho(p, e, k) .
$$

We prove (1), (2) and (3) by induction on $e$.

If $e=1$ then $y_{0} p+y_{1}=k=a_{1} p+a_{0}$. Since $0 \leq a_{0}<p, y_{1}=a_{0}+z_{1} p$ with $z_{1} \geq 0$, so that $y_{0}=a_{1}-z_{1}$. Hence $\delta_{1}(y, k)=z_{1}(p-1) \geq 0$. Also $y_{1} \neq a_{0}$ if and only if $y_{0} \neq a_{1}$ if and only if $z_{1} \geq 1$. So (1) and (2) hold for $e=1$.

Now suppose $k=\sum_{j=0}^{e+1} a_{j} p^{j}=\sum_{i=0}^{e+1} y_{i} p^{e+1-i}$. Let $k_{1}=(1 / p)\left(k-a_{0}\right)=\sum_{i=0}^{e} b_{t} p^{t}$, where $0 \leq b_{t}=a_{t+1}<p$ for $t=0,1, \ldots, e-1$ and $b_{e}=a_{e+1} \geq 0$. As above, $y_{e+1}=a_{0}+z_{e+1} p$ with $z_{e+1}$ a non-negative integer. Now $k_{1}=\sum_{i=0}^{e} y_{i}^{\prime} p^{e-1}$ where $y_{i}^{\prime}=y_{i}$ for $i=0,1, \ldots, e-1$ and $y_{e}^{\prime}=y_{e}+z_{e+1}$. Thus $\delta_{e+1}(y, k)=\delta_{e}\left(y^{\prime}, k_{1}\right)+z_{e+1}(p-1) \geq 0$ by induction. In addition, if 
$y_{e+1} \neq a_{0}$, then $z_{e+1}>0$ so that (2) also holds. If $y_{e+1}=a_{0}$, then $z_{e+1}=0$ and $y_{e}^{\prime}=y_{e}$. So, if $y_{i} \neq a_{e+1-i}$ for some $0 \leq i \leq e$, then $y_{i}^{\prime} \neq b_{e-1}$ and again by induction

$$
\delta_{e+1}(y, k)=\delta_{e}\left(y^{\prime}, k\right) \geq p-1 .
$$

When $p=2, \delta_{1}(y, k)=z_{1}=\frac{1}{2}\left(y_{1}-a_{0}\right)$. Using induction as above we have

$$
\delta_{e+1}(y, k)=\delta_{e}\left(y^{\prime}, k_{1}\right)+z_{e+1} \geq \frac{1}{2}\left(y_{i}^{\prime}-b_{e-1}\right)+z_{e+1}=\frac{1}{2}\left(y_{1}-a_{e}\right)+z_{e+1}
$$

which proves (3).

Finally, for (4), if $k$ is a multiple of 4 and $e>1$, then either $k=a_{e} 2^{e}$ with $a_{e} \geq 2$ or $k=2^{t}+\sum_{j=t+1}^{e} a_{j} 2^{j}$ for some $2 \leq t \leq e$. So,

$$
k-2=\left(y_{e}-2\right)+\sum_{i=0}^{e-1} y_{i} 2^{e-i}=\left(a_{e}-1\right) 2^{e}+\sum_{j=1}^{e-1} 2^{j} \text { or } \sum_{j=t+1}^{e} a_{j} 2^{j}+\sum_{j=1}^{t-1} 2^{j}
$$

Thus by (1), $y_{e}-2+\sum_{i=0}^{e-1} y_{i} \geq\left(a_{e}-1\right)+(e-1)$ or $\sum_{j=t+1}^{e} a_{j}+(t-1)$. Hence $\delta_{e}(y, k) \geq t \geq 2$ in both cases.

REMARK. (4) above is not necessarily true without the requirement that $k$ be a multiple of 4.

The following technical corollary will be used in computing $p_{-}\left(2^{e}, k\right)$.

Corollary 1.2. Let $k \geq 1, e \geq 2$ be integers. Let $y_{0}, \ldots, y_{e}, \alpha$ be non-negative integers such that $k=\alpha 2^{e-1}+\sum_{i=0}^{e} y_{i} 2^{e-i}$. Then, if $q=\sum_{i=0}^{e} y_{i}, 2 q+\alpha \geq \varepsilon(e, k)$.

Proof. By definition, $\varepsilon(e, k)=b_{e-1}+2 \sum_{j=0}^{e-2} b_{j}$ with $k=\sum_{j=0}^{e-1} b_{j} 2^{j}$ and $0 \leq b_{j} \leq 1 \quad(j=$ $0,1, \ldots, e-2), b_{e-1} \geq 0$. Let us divide $b_{e-1}=2 b_{e}+c, 0 \leq c \leq 1$, and so

$$
b_{e} 2^{e}+c 2^{e-1}+\sum_{j=0}^{e-2} b_{j} 2^{j}=k=y_{0} 2^{e}+\left(y_{1}+\alpha\right) 2^{e-1}+\sum_{i=2}^{e} y_{i} 2^{e-i}
$$

From (3) in Lemma 1.1, $\alpha+\sum_{i=0}^{e} y_{i}-\left(b_{e}+c+\sum_{j=0}^{e-2} b_{j}\right) \geq\left(y_{1}+\alpha-c\right) / 2$, i.e.,

$$
2 \alpha+2 q-2 \sum_{j=0}^{e-2} b_{j} \geq 2\left(b_{e}+c\right)+y_{1}+\alpha-c=b_{e-1}+y_{1}+\alpha \geq b_{e-1}+\alpha
$$

Therefore, $2 q+\alpha \geq b_{e-1}+2 \sum_{j=0}^{e-2} b_{j}=\varepsilon(e, k)$.

2. The computation of $p_{+}^{+}\left(p^{e}, k\right)$. As stated in the introduction, the case $e=1$ is solved in [4; Cor 3.2.17] and so we assume $e \geq 2$. Thus $p^{e}$ is a multiple of 4 if $p=2$ and so, from Theorems 3.2.3, 3.1.2 and 3.1.5 (this last ot cover the case $p=2$ ) in [4], the 
existence of a surface $S$ in $K_{+}^{+}\left(p^{e}, k\right)$ with topological genus $g=g(S)$ is equivalent to the existence of non-negative integers $g^{\prime}, m_{1}, \ldots, m_{r}, t_{1}, \ldots, t_{k^{\prime}}$ with $m_{i} \geq 2$ and $m_{i}, t_{j}$ divisors of $p^{e}$ such that

$$
\begin{gathered}
\mu=2 g^{\prime}+k^{\prime}-2+\sum_{i=1}^{r}\left(1-\frac{1}{m_{i}}\right)>0 ; \quad 2(g-1)+k=p^{e} \mu \\
k=\sum_{j=1}^{k^{\prime}} \frac{p^{e}}{t_{j}} \\
\text { If } g^{\prime}=0 \text {, then l.c.m. }\left\{m_{1}, \ldots, m_{r}, t_{1}, \ldots, t_{k^{\prime}}\right\}=p^{e} .
\end{gathered}
$$

(2.4) The set $X=\left\{m_{1}, \ldots, m_{r}, t_{1}, \ldots, t_{k^{\prime}}\right\}$ has the elimination property, i.e., the l.c.m. does not change if we delete one of these numbers, and so, in particular, if $X$ has just one element, then $X=\{1\}$.

Note that from $(2.1), p^{e} \mu=2(g-1)+k=p(S)-1$, and so our task is to minimize the value of $\mu$ constrained to conditions (2.1), (2.2), (2.3) and (2.4). The cases $k=1$ and $k=2$ are easier, but they need separate arguments. So we begin with

First case: $k=1$. Then necessarily $k^{\prime}=1, t_{1}=p^{e}$, condition (2.3) is automatically satisfied and from (2.4), $r \geq 1, m_{1}=p^{e}$. Since $\mu$ must be positive it is obvious that its minimum value is attained for

$$
g^{\prime}=0, \quad k^{\prime}=1, \quad t_{1}=p^{e}, \quad r=2, \quad m_{1}=p^{e}, \quad m_{2}=p
$$

and for this choice, $\mu=1-\frac{1}{p^{e}}-\frac{1}{p}$. Since $p(S)=1+p^{e} \mu$ we obtain $p_{+}^{+}\left(p^{e}, 1\right)=p^{e}-p^{e-1}$.

Second case: $k=2$. Here we distinguish two subcases: $p$ is odd or $p=2$. For odd $p$, the divisors $\frac{p^{e}}{t_{j}}$ of $p^{e}$ are odd and so, by (2.2), $k^{\prime}=2, t_{1}=t_{2}=p^{e}$. This way conditions (2.3) and (2.4) are satisfied for any choice of $m_{1}, \ldots, m_{r}$. Hence, the minimum value of $\mu$ is attained for

$$
g^{\prime}=0, \quad k^{\prime}=2, \quad t_{1}=t_{2}=p^{e}, \quad r=1, \quad m_{1}=p
$$

and then $\mu=1-\frac{1}{p}$, i.e., $p_{+}^{+}\left(p^{e}, 2\right)=p^{e}-p^{e-1}+1$ if $p$ is odd.

If $p=2$, a new possibility occurs: $k^{\prime}=1, t_{1}=2^{e-1}$. The corresponding value of $\mu$ for $g^{\prime}>0$ is bigger than or equal to $1>1-\frac{1}{2}$, and so we are restricted to analyze the choices with $g^{\prime}=0$. Condition (2.3) imposes $r \geq 1, m_{1}=2^{e}$, and from (2.4), $r \geq 2$ and $m_{2}=2^{e}$. Hence $\mu \geq-1+2\left(1-\frac{1}{2^{e}}\right) \geq 1-\frac{1}{2}$. Therefore,

$$
p_{+}^{+}\left(p^{e}, 2\right)=p^{e}-p^{e-1}+1 \text { for all } p .
$$

For the remainder of this secton, we assume that $k>2$. First, we reformulate conditions (2.1)-(2.4), and the notation now introduced will be used consistently throughout the paper. Since each $m_{i}, t_{j}$ is a power of $p$, we define $x_{i}=\operatorname{Card}\left\{m_{s}: m_{s}=p^{i}\right\}, 1 \leq i \leq e$; $y_{i}=\operatorname{Card}\left\{t_{s}: t_{s}=p^{i}\right\}, 0 \leq i \leq e$. This data is denoted by $F=\left\{x_{i}, y_{j}: 1 \leq i \leq e, 0 \leq j \leq e\right\}$.

$$
\text { Let } \Lambda_{F}=\left\{i: 1 \leq i \leq e, x_{i}+y_{i} \neq 0\right\} \quad \text { and } \quad M_{F}= \begin{cases}0 & \text { if } \Lambda_{F} \text { is empty } \\ \max \Lambda_{F} & \text { otherwise. }\end{cases}
$$


Now $k^{\prime}=\sum_{i=0}^{e} y_{i}$ and conditions (2.1)-(2.4) can be stated as:

$$
\begin{gathered}
\mu=2\left(g^{\prime}-1\right)+\sum_{i=0}^{e} y_{i}+\sum_{i=1}^{e} x_{i}\left(1-\frac{1}{p^{i}}\right)>0 ; \quad p(S)-1=2(g-1)+k=p^{e} \mu . \\
k=\sum_{i=0}^{e} y_{i} p^{e-i} . \\
\text { If } g^{\prime}=0, \text { then } M_{F}=e\left(\text { i.e., } x_{e}+y_{e} \geq 1\right) . \\
\text { Either } M_{F}=0 \text { or } x_{M_{F}}+y_{M_{F}} \geq 2 .
\end{gathered}
$$

Hence, our problem is to choose $g^{\prime}$ and the set $F$ in such a way that $\mu$ is minimal and these "new" conditions (2.1)-(2.4) are satisfied. We are led to consider different cases:

Third case: $k>2, k \neq 0,1(\bmod p)$. For any choice of $y_{0}, \ldots, y_{e}, k \equiv y_{e}(\bmod p)$, and so $y_{e} \geq 2$, which guarantees that conditions (2.3) and (2.4) are satisfied for any choice of $x_{1}, \ldots, x_{e}$. Moreover, since $k>2$ and $y_{e} \geq 2$ then $\sum_{i=0}^{e} y_{i} \geq 3$ and so $\mu>0$ for any choice of $g^{\prime}$. Hence, the minimum value of $\mu$ shall be obtained for $g^{\prime}=0, x_{1}=\ldots=x_{e}=0$ and $\sum_{i=0}^{e} y_{i}$ minimal satisfying (2.2). Therefore, choosing $y_{i}=a_{e-i}(p, e, k)$ we conclude from part (1) in Lemma 1.1 that the minimum value of $\mu$ is $\rho(p, e, k)-2$. Consequently,

$$
p_{+}^{+}\left(p^{e}, k\right)=p^{e}[\rho(p, e, k)-2]+1 \text {. }
$$

Fourth case: $k>2, k \equiv 1(\bmod p)$. Consider our "canonical" $p$-adic expansion $k=$ $\sum_{j=0}^{e} a_{j} p^{j}$ where $a_{j}=a_{j}(p, e, k)$. We must have $a_{0}=1$, and thus, by choosing

$$
g^{\prime}=0, \quad x_{1}=\ldots=x_{e-1}=0, \quad x_{e}=1, \quad y_{i}=a_{e-i}, \quad 0 \leq i \leq e
$$

we obtain the required conditions (2.1)-(2.4) (since $x_{e}=y_{e}=1$ and $\mu_{0}=-2+$ $\rho(p, e, k)+1-\frac{1}{p^{e}}$ is positive $)$. We are going to prove this is the minimum value of $\mu$. Let $\left\{g^{\prime}, x_{1}, \ldots, x_{e}, y_{0}, \ldots, y_{e}\right\}$ be another choice satisfying the restrictions. If $y_{e} \geq 2$, then by part (2) in Lemma 1.1, $\sum_{i=0}^{e} y_{i} \geq \rho(p, e, k)+p-1$ and therefore the corresponding value of $\mu$ satisfies

$$
\mu \geq-2+\sum_{i=0}^{e} y_{i} \geq \rho(p, e, k)+p-3 \geq \rho(p, e, k)-1>\mu_{0} .
$$

On the other hand, if $y_{e}<2$ then $y_{e}=1$ since $k \equiv 1(\bmod p)$. Thus $M_{F}=e$ and by (2.4), $x_{e} \geq 1$. By part (1) in Lemma 1.1, $\mu \geq-2+\sum_{i=0}^{e} y_{i}+1-\frac{1}{p^{e}} \geq \mu_{0}$. Hence

$$
p_{+}^{+}\left(p^{e}, k\right)=p^{e}[\rho(p, e, k)-1] \text {. }
$$

Fifth case: $k>2, k \equiv 0(\bmod p), p$ odd. As before we take the " $p$-adic" expansion of $k, k=\sum_{j=0}^{e} a_{j} p^{j}, 0 \leq a_{j}<p,(j=0,1, \ldots, e-1)$, and $a_{e} \geq 0$. Of course $a_{0}=0$ and the choice 
$g^{\prime}=0 ; x_{1}=\ldots=x_{e-1}=0 ; y_{i}=a_{e-i}, 0 \leq i \leq e$, gives the value $\mu_{0}=\frac{-2}{p^{e}}+\rho(p, e, k)>0$ since $\sum_{j=0}^{e} a_{j} \geq 1$ as $k \neq 0$. Since $x_{e}=2$, this is an admissible choice, and it is easy to check that it is the best one.

Consequently $p_{+}^{+}\left(p^{e}, k\right)=p^{e} \rho(p, e, k)-1$.

To finish this section we only need to study:

Sixth case: $k>2, k \equiv 0(\bmod 2), p=2$. Now we must distinguish two subcases: $k$ is multiple of 4 or not. In the first one we can repeat the argument of the previous case word for word using part (4) of Lemma 1.1 instead of part (2).

Note that only the case $y_{e} \neq 0$ requires some comment but then $y_{e} \geq 2$ since $k$ is even, and so $\sum_{i=0}^{e} y_{i}-\rho(2, e, k) \geq 2$ which provides, for the corresponding $\mu$, the inequality $\mu \geq-2+\sum_{i=0}^{e} y_{i} \geq \rho(2, e, k)>\mu_{0}=\rho(2, e, k)-\frac{2}{2^{e}}$. Hence $p_{+}^{+}\left(2^{e}, k\right)=2^{e} \rho(2, e, k)-1$ if $k$ is multiple of 4 .

Suppose now $k$ is not a multiple of 4 , and so its " $p$-adic" expansion is $k=\sum_{j=0}^{e} a_{j} 2^{j}$, $a_{1}=1,0 \leq a_{j} \leq 1,(j=2, \ldots, e-1)$ and $a_{e} \geq 0$. This time we choose $g^{\prime}=0, x_{1}=\ldots=x_{e}=$ $0, y_{i}=a_{e-1}, 0 \leq i \leq e-2, y_{e-1}=0, y_{e}=2$. This is an admissible choice since $y_{e}=2$ and the corresponding value of $\mu$ is given by $\mu_{0}=-2+\sum_{i=0}^{e} y_{i}=\rho(2, e, k)-1$, which is positive since $a_{1}=1$ and $k>2$. It is straightforward to check that $\mu_{0}$ is the minimum value of $\mu$ and therefore $p_{+}^{+}\left(2^{e}, k\right)=2^{e}[\rho(2, e, k)-1]$ if $k$ is not a multiple of 4 .

The results in this section, together with Cor 3.2.17 in [4] for the case $e=1$, can be summarized as follows:

THEOREM 1. Let $k, e$ be positive integers and let $p$ be a prime number. Let $k=\sum_{j=0}^{e} a_{j} p^{j}$ with $0 \leq a_{j}<p$ for $j=0, \ldots, e-1$ and $a_{e} \geq 0$. Let $\rho(p, e, k)=\sum_{j=0}^{e} a_{j}$. Then

$$
p_{+}^{+}\left(p^{e}, k\right)=\left\{\begin{array}{lll}
2 & k=1, \quad e=1, \quad p=2 \\
p^{e}-p^{e-1} & k=1, \quad(e, p) \neq(1,2) \\
3 & k=2, \quad(e, p)=(1,2) \\
p^{e}-p^{e-1}+1 & k=2, \quad(e, p) \neq(1,2) \\
p^{e}[\rho(p, e, k)-2]+1 & k>2, k \neq 0,1(\bmod p) \\
p^{e}[\rho(p, e, k)-1] & k>2, k \equiv 1(\bmod p) \\
2^{e}[\rho(p, e, k)-1]+1 & k>2, k \equiv 2(\bmod 4), \quad p=2, e>1 \\
p^{e} \rho(p, e, k)-1 & \text { otherwise }
\end{array}\right.
$$

We note that for $p$ odd, the minimum genus of a complex algebraic curve admitting an automorphism of order $p^{e}$ is $1 / 2\left(p^{e}-p^{e-1}\right)$ [7]. It is interesting to compre this with the results for $g_{+}^{+}$obtained from Theorem 1 . Note that $g_{+}^{+}=0$ for $k$ of the form $a_{e} p^{e}+a_{0}$ 
where $a_{0}=0,1,2$, which will, of course, correspond to rotations of the sphere. Otherwise $g_{+}^{+} \geq 1 / 2\left(p^{e}-p^{e-1}\right)$. Note that the values of $k$ for which we get equality here have the form $a_{e} p^{e}+p^{e^{-1}}+a_{0}$ where $a_{0}=0,1,2$.

3. The computation of $p_{+}^{-}\left(p^{e}, k\right)$. The family $K_{+}^{-}(N, k)$ is empty for odd $N-$ see [4; Prop. 3.2.3] and so we are only concerned with the calculus of $p_{+}^{-}\left(2^{e}, k\right)$. As above, we assume $e \geq 2$. Using Thms 3.1.9 and 3.2.3 in [4], and since $2^{e}$ is multiple of 4 , the existence of a surface $S$ in $K_{+}^{-}\left(2^{e}, k\right)$ with topological genus $g=g(S)$ is equivalent to the existence of non-negative integers $g^{\prime} \geq 1, m_{1}, \ldots, m_{r}, t_{1}, \ldots, t_{k^{\prime}}$ with $m_{i} \geq 2$ and $m_{i}, t_{j}$ divisors of $2^{e-1}$ such that

$$
\mu=g^{\prime}+k^{\prime}-2+\sum_{i=1}^{r}\left(1-\frac{1}{m_{i}}\right)>0 ; p(S)-1=2(g-1)+k=2^{e} \mu \text {. }
$$

$$
k=\sum_{j=1}^{k^{\prime}} \frac{2^{e}}{t_{j}}
$$

(3.3) If $g^{\prime}=1$, then l.c.m. $\left\{m_{1}, \ldots, m_{r}, t_{1}, \ldots, t_{k^{\prime}}\right\}=2^{e-1}$.

(3.4) There exist $\alpha_{1}, \ldots, \alpha_{r}, \beta_{1}, \ldots, \beta_{k^{\prime}}$ with g.c.d. $\left(\alpha_{i}, m_{i}\right)=1=$ g.c.d. $\left(\beta_{j}, t_{j}\right)$ for every $i$ and $j$ such that $g^{\prime}$ is even if and only if $M=\sum_{i=1}^{r} \alpha_{i} \frac{2^{e}}{m_{i}}+\sum_{j=1}^{k^{\prime}} \beta_{j} \frac{2^{e}}{t_{j}}$ is a multiple of 4 .

Let us analyze more carefully condition 3.4. As before, write

$$
x_{i}=\operatorname{Card}\left\{m_{s}: m_{s}=2^{i}\right\}, \quad 1 \leq i \leq e-1 ; \quad y_{i}=\operatorname{Card}\left\{t_{s}: t_{s}=2^{i}\right\}, \quad 0 \leq i \leq e-1 .
$$

Since each $m_{i}$ is even, $\alpha_{i}=2 \gamma_{i}+1,1 \leq i \leq r$, and so

$$
\sum_{i=1}^{r} \alpha_{i} \frac{2^{e}}{m_{i}} \equiv \sum_{i=1}^{r} \frac{2^{e}}{m_{i}} \equiv 2 x_{e-1}(\bmod 4) .
$$

On the other hand, $\beta_{j}=2 \delta_{j}+1$ if $t_{j} \neq 1$ and so,

$$
\sum_{j=1}^{k^{\prime}} \beta_{j} \frac{2^{e}}{t_{j}} \equiv \sum_{j=1 ; t_{j} \neq 1}^{k^{\prime}} \frac{2^{e}}{t_{j}} \equiv 2 y_{e-1}(\bmod 4)
$$

Therefore, $M \equiv 2\left(x_{e-1}+y_{e-1}\right)(\bmod 4)$. Hence, the conditions above can be expressed as:

$$
\begin{gathered}
\mu=g^{\prime}-2+\sum_{i=0}^{e-1} y_{i}+\sum_{i=1}^{e-1} x_{i}\left(1-\frac{1}{2^{i}}\right)>0 ; \quad p(S)=2^{e} \mu+1 \\
k=\sum_{i=0}^{e-1} y_{i} 2^{e-i} \\
\text { If } g^{\prime}=1, \text { then } x_{e-1}+y_{e-1} \geq 1 \\
g^{\prime} \text { is even if and only if } x_{e-1}+y_{e-1} \text { is even. }
\end{gathered}
$$

In particular we obtain the following interesting result.

Proposition 3.1. Let $S$ be an orientable compact Klein surface of algebraic genus $g \geq 2$, whose boundary has an odd number of connected components. Then, every automorphism of $S$ whose order is a multiple of 4 , preserves the orientation of $S$.

Proof. Let $f$ be an automorphism on $S$ of order $N=2^{e} M$ with $e \geq 2$ and odd $M$, and assume that the number $k$ of connected components of the boundary of $S$ is odd. If $f$ 
reverses the orientation of $S$ the same holds true for $g=f^{M}$, which has order $2^{e}, e \geq 2$. But then, by condition (3.2) above, $k$ must be even.

In other words, $K_{+}^{-}\left(2^{e}, k\right)$ is empty for odd $k$ and $e \geq 2$. Hence we assume throughout this section that $k$ is even. The arguments here are somewhat similar to those used in the preceding and consequently we only outline them.

First case: $k=2$. From (3.2) we must have $y_{e-1}=1, y_{0}=\ldots=y_{e-2}=0$ and so $\mu=g^{\prime}-1+\sum_{i=1}^{e-1} x_{i}\left(1-\frac{1}{2^{i}}\right)$. If $e=2$, the choice $g^{\prime}=1, x_{1}=2$ satisfies (3.1)-(3.4) and is easily shown to give the minimum value $\mu_{0}=1$ of $\mu$. Hence $p_{+}^{-}(4,2)=5$. Similarly, if $e>2$, taking $g^{\prime}=1, x_{1}=1, x_{i}=0,2 \leq i \leq e-1$ achieves the minimum giving $p_{+}^{-}\left(2^{e}, 2\right)=$ $2^{e-1}+1$ if $e>2$.

Second case: $k=0(\bmod 4)$. Let $R=\frac{k}{2}$ have canonical " 2 -adic" expansion $R=\sum_{j=0}^{e-1} a_{j} 2^{j}$ with $0 \leq a_{j} \leq 1$ for $0 \leq j \leq e-2$ and $a_{e-1} \geq 0$. This time the choice $g^{\prime}=1, y_{i}=a_{(e-1)-i}$ for $0 \leq i \leq e-1, x_{1}=\ldots=x_{e-2}=0, x_{e-1}=1$ can be shown to give the minimum value of $\mu$ using (1) in Lemma 1.1 applied to $R$. Thus

$$
p_{+}^{-}\left(2^{e}, k\right)=2^{e} \rho\left(2, e-1, \frac{k}{2}\right)-1 \quad \text { for } \quad k \equiv 0(\bmod 4) \text {. }
$$

Third case: $k>2, k=2(\bmod 4)$. As above let $R=\frac{k}{2}=\sum_{j=0}^{e-1} a_{j} 2^{j}$ in canonical form. The minimal data here is $g^{\prime}=1, y_{i}=a_{(e-1)-i}$ for $0 \leq i \leq e-1, x_{i}=0$ for $1 \leq i \leq e-1$ giving $\mu=-1+\sum_{i=0}^{e-1} a_{i}>0$ since $a_{0}=1$ and $R>1$. Therefore,

in this situation.

$$
p_{+}^{-}\left(2^{e}, k\right)=2^{e}\left[\rho\left(2, e-1, \frac{k}{2}\right)-1\right]+1
$$

Including the cases where $e=1$ for completeness from Theorem 3.2.17 in [4] we obtain

THEOREM 2. Let $k$, e be positive integers and let $p$ be a prime number. Then, $K_{+}^{-}\left(p^{e}, k\right)$ is empty if either $p$ is odd or $p=2, e \geq 2$, and $k$ is odd.

$$
p_{+}^{-}\left(2^{e}, k\right)= \begin{cases}k-1 & e=1, k>2 \\ k+1 & e=1, k \leq 2 \\ 5 & e=k=2 \\ 2^{e-1}+1 & e>2, k=2 \\ 2^{e} \rho\left(2, e-1, \frac{k}{2}\right)-1 & e \geq 2, k \equiv 0(\bmod 4) \\ 2^{e}\left[\rho\left(2, e-1, \frac{k}{2}\right)-1\right]+1 & e \geq 2, k>2, k \equiv 2(\bmod 4) .\end{cases}
$$

4. The computation of $p_{-}\left(p^{e}, k\right)$ for odd prime $p$. Let $e \geq 1$. With the usual notation, Theorem 3.1.3 in [4] shows that the existence of a surface $S$ in $K_{-}\left(p^{e}, k\right)$ with topological genus $g=g(S)$ is equivalent to the existence of non-negative integers $g^{\prime} \geq 1$, 
$x_{1}, \ldots, x_{e}, y_{0}, \ldots, y_{e}$ such that:

$$
\begin{aligned}
& \begin{array}{l}
\mu=g^{\prime}-2+\sum_{i=0}^{e} y_{i}+\sum_{i=1}^{e} x_{i}\left(1-\frac{1}{p^{i}}\right)>0, \quad p(S)=2(g-1)+k+1=p^{e} \mu+1 . \\
k=\sum_{i=0}^{e} y_{i} p^{e-i}
\end{array} \\
& \text { If } g^{\prime}=1, \text { then } x_{e}+y_{e} \geq 1 .
\end{aligned}
$$

The arguments involved in the computation of $p_{-}\left(p^{e}, k\right)$ are now very similar to those in Section 2 and the details can be easily completed. The results are given in Theorem 3 at the end of Section 5.

5. The computation of $p_{-}\left(2^{e}, k\right), e \geq 2$. This case is more complicated as surfaces $S$ in $K_{-}\left(2^{e}, k\right)$ with topological genus $g=g(S)$, can arise in two ways as given by Theorem 3.1.6 or Theorem 3.1 .8 in [4].

This has an obvious explanation, since we are lead to different conditions according to the quotient of $S$ under the involved automorphism being orientable or not.

In fact, it turns out that the minimum genus always corresponds to a solution of the conditions given by Theorem 3.1.6, so we first reformulate these conditions.

Setting $t=k^{\prime}-p^{\prime}$, and $\alpha=\sum_{i=s^{\prime}+1}^{k^{\prime}} \frac{r_{i}^{\prime}}{2}$ in Theorem 3.1.6, it will follow that such a surface $S$ will occur if and only if there exist non-negative integers $g^{\prime}, t>0, x_{1}, \ldots, x_{e}$, $y_{0}, \ldots, y_{e}, \alpha$ such that:

$$
\begin{aligned}
& \mu=2\left(g^{\prime}-1\right)+t+\sum_{i=0}^{e} y_{i}+\sum_{i=1}^{e} x_{i}\left(1-\frac{1}{2^{i}}\right)+\frac{\alpha}{2}>0 ; \quad p(S)=g(S)+k-1=2^{e} \mu+1 . \\
& k=\sum_{i=0}^{e} y_{i} 2^{e-i}+\alpha 2^{e-1} \\
& \text { If } g^{\prime}=0 \text { and } t=1 \text {, then } x_{e+} y_{e} \geq 1 .
\end{aligned}
$$

It is convenient to use $\delta=2 \mu$, so that

$$
\delta=4\left(g^{\prime}-1\right)+2 t+\sum_{i=1}^{e} 2 x_{i}\left(1-\frac{1}{2^{i}}\right)+\left[2 \sum_{i=0}^{e} y_{i}+\alpha\right]>0, \quad \text { and } \quad p(S)=1+e^{e-1} \delta
$$

Note, from (5.2) and the term in square brackets above, that this is precisely the situation in which we can apply Corollary 1.2.

Using this Corollary and notation of Section 1, variations of minimizing arguments above yield the following minimum values for $\delta$.

(i) $\delta_{0}=1$ if $k=1$.

(ii) $\delta_{0}=-2+\varepsilon(e, k)$ if $k \equiv 1(\bmod 2), k>1$.

(iii) $\delta_{0}=-2^{-(e-1)}+\varepsilon(e, k)$ if $k \equiv 0(\bmod 2), k>1$.

Now we reformulate the conditions contained in Theorem 3.1.8 of [4] as follows: 
there exist non-negative integers $g^{\prime} \geq 1, t \geq 0, \alpha, x_{1}, \ldots, x_{e}, y_{0}, \ldots, y_{e}$ such that:

$$
\begin{gathered}
\mu=g^{\prime}-2+t+\sum_{i=0}^{e} y_{i}+\sum_{i=1}^{e} x_{i}\left(1-\frac{1}{2^{i}}\right)+\frac{\alpha}{2}>0 ; \quad p(S)=g(S)+k-1=2^{e} \mu+1 . \\
k=\sum_{i=0}^{e} y_{i} 2^{e-i}+\alpha 2^{e-1} \\
\text { If } t=0 \text {, then } \alpha=0 \text { and } x_{e}+y_{e} \text { is even. } \\
\text { If } t=0 \text { and } g^{\prime}=1 \text { then } x_{e}+y_{e} \geq 1 . \\
\text { If } t=0, g^{\prime}=2 \text { and } \sum_{i=e-1}^{e}\left(x_{i}+y_{i}\right) 2^{e-i} \equiv 0(\bmod 4) \text {, then } x_{e}+y_{e} \geq 1 .
\end{gathered}
$$

In each of the cases studied above, we find that the minimum value $\delta_{0}$ of $\delta$ obtained from conditions (5.1)-(5.3) is smaller than all values of $\delta$ obtained from parameters satisfying conditions (5.4)-(5.8). The arguments to establish this follow the same lines as those given, and are left to the reader.

Summarizing the results obtained in this and the preceding section and also the cases where $e=1$ contained in Theorem 3.2 .15 of [4] we obtain:

THEOREM 3. Let $k, e$, be positive integers and let $p$ be a prime number. Then

$$
p_{-}\left(p^{e}, k\right)= \begin{cases}(p-1) p^{e-1}+1 & k=1 \\ p^{e}[\rho(p, e, k)-1]+1 & k>1, p \text { odd }, k \neq 0(\bmod p) \\ p^{e} \rho(p, e, k) & p \text { odd }, k \equiv 0(\bmod p) \\ k & k>1, p=2, e=1 \\ 2^{e-1}[\varepsilon(e, k)-2]+1 & k>1, k \text { odd }, p=2, e>1 \\ 2^{e-1} \varepsilon(e, k) & k \text { even }, p=2, e>1 .\end{cases}
$$

Theorems 1, 2 and 3 give a complete solution to the problem of calculating the numbers $p_{+}^{+}(N, k), p_{+}^{-}(N, k)$ and $p_{-}(N, k)$ when $N$ is a power of a prime number. Taking into account that bordered and compact Klein surfaces are simply projective, smooth, irreducible real algebraic curves, so that if $C$ is the curve associated with the surface $S$, then $C$ is homeomorphic to the boundary of $S$ and the surface $S$ is orientable if and only if the curve $C$ disconnects its complexification, our results can be stated in terms of birational automorphisms of real projective, smooth, irreducible algebraic curves with fixed number of connected components, as was observed in the introduction.

Note that it is a routine matter to determine the constants $\rho$ and $\varepsilon$ in which our results are stated from the truncated $p$-adic expansions.

ExAmple. Take $p^{e}=16=2^{4}$ and $k=54$. Then

$$
k=2+2^{2}+3 \cdot 2^{4}=2+2^{2}+6 \cdot 2^{3} \text {. }
$$

Thus $\rho=\rho(2, e, k)=5=\rho(2, e-1, k / 2) ; \varepsilon=\varepsilon(e, k)=10$. Thus, from Theorems 1,2 and 3 we obtain

$$
p_{+}^{+}(16,54)=65=p_{+}^{-}(16,54) ; \quad p_{-}(16,54)=80 \text {. }
$$

Thus from these we obtain in these cases that

$$
g_{+}^{+}=6=g_{+}^{-} ; \quad g_{-}=27
$$




\section{REFERENCES}

1. N. L. Alling and N. Greenleaf, Foundations of the theory of Klein surfaces . Lecture Notes in Mathematics No 219 (Springer-Verlag, 1971).

2. E. Bujalance, Cyclic groups of automorphisms of compact non-orientable surfaces without boundary. Pacific J. Math. 109 (1983), 279-289.

3. E. Bujalance, J. J. Etayo, J. M. Gamboa and G. Martens, Minimal genus of Klein surfaces admitting an automorphism of given order. Arch. Math. 52 (1989) 191-202.

4. E. Bujalance, J. J. Etayo, J. M. Gamboa and G. Gromadzki, Automorphism Groups of Compact Bordered Klein Surfaces. A combinatorial approach. Lecture Notes in Mathematics No 1439 (Springer-Verlag, 1990).

5. G. Gromadzki, Abelian groups of automorphisms of compact non-orientable Klein surfaces without boundary, Commentationes Math. 28 (1989), 197-217.

6. W. Hall, Automorphisms and coverings of Klein surfaces. Ph.D. Thesis, Southampton University (1977).

7. W. J. Harvey, Cyclic groups of automorphisms of a compact Riemann surface. Quart. J. Math. Oxford (2) 17 (1966), 86-97.

8. A. Hurwitz, Über algebraische Gebilde mit eindeutigen Transformationen in sich, Math. Ann. 41 (1893), 402-442.

9. R. S. Kulkarni and C. Maclachlan, Cyclic p-groups of symmetries of surfaces. Glasgow Math. J. 33 (1991), 213-221.

10. C. Maclachlan, Abelian groups of automorphisms of compact Riemann surfaces, Proc. London Math. Soc. 15 (1965), 600-712.

11. C. L. May, Automorphisms of compact Klein surfaces with boundary, Pacific J. Math. 59 (1975), 199-210.

12. C. L. May, Cyclic automorphism groups of compact bordered Klein surfaces, Houston J. Math. 3 (1977), 395-405.

13. D. McCullough, Minimal genus of abelian actions on Klein surfaces with boundary, Math. Zeit. 205 (1990), 421-436.

14. A. Wiman, Über die hyperelliptischen Kurven und diejenigen von Geschlechte $p=3$, welche eideutigen Trasformationen in sich zulassen. Bihang Kongl. Svenska Vetenskaps-Akademiens Handlingar (Stockholm, 1895-96).

EMILIO BuJaLANCE

Departamento de Matemáticas Fundamentales

FaC. Ciencias

U.N.E.D.

28040 MADRID

SPAIN

José Manuel Gamboa

Departamento de Algebra

FaC. C. Matemáticas

UNIVERSIDAD COMPLUTENSE

28040 MADRID

SPAIN

Colin Maclachlan

Department of Mathematics

UNIVERSITY OF ABERDEEN

ABERDEEN AB9 2TY

SCOTLAND 